\title{
Anemia en mujeres en edad fértil de la Comunidad Nativa Ese'eja - Palma Real, Madre Dios, Perú
}

\begin{abstract}
Anemia in women of childbearing age in the Native Community Ese'eja-- Palma Real, Madre Dios, Peru
J. Antonio Grandez-Urbina ${ }^{1,2,3, a}$, Gabriela Cervantes-Siles ${ }^{1, a}$, Jorge Castro-Segura ${ }^{1,2, a}$, Diana LlactaAparicio $^{1,2, b}$, J. Gonzalo Rodríguez ${ }^{1,2, a}$.

\section{RESUMEN}

Objetivos: Determinar la prevalencia de anemia en mujeres en edad fértil en comunidades nativas Ese'eja en Madre de Dios, Perú. Material y métodos: Estudio observacional, descriptivo y transversal, un muestreo tipo censal, se determinó la concentración de hemoglobina mediante la técnica de micro hematocrito. El análisis de los datos se realizó con el paquete estadístico STATA 11.0 (STATA Corp. Texas, US). Resultados: El 77,63\% de las mujeres mostró cifras de hemoglobina inferiores a $12 \mathrm{~g} / \mathrm{dl}$ de ellas, el 29,5\% se encontraba entre 11,0 y 11,9 g/dl, lo que se considera como anemia leve; el 70,5\% restante tenía la hemoglobina entre 7,0 y 10,9 g/dl. Conclusiones: La anemia es una alteración altamente prevalente en mujeres en edad fértil de la comunidad nativa Ese'eja Palma Real.
\end{abstract}

PALABRAS CLAVE: Anemia, deficiencia de hierro, salud de la mujer, salud pública, atención prenatal. (Fuente: DeCS BIREME)

\section{SUMMARY}

Objectives: To determine the prevalence of anemia in women of childbearing age in the Ese'eja native communities. Methods: An observational, descriptive and transversal study with census sampling. The hemoglobin was determined by micro hematocrit technique. Data analysis was performed using the statistical package STATA 11.0 (Stata Corp. Texas, U.S.). Results: Hemoglobin levels below $12 \mathrm{~g} / \mathrm{dl}$ was recorded in $77.63 \%$ of women, $29.5 \%$ of them had mild anemia (hemoglobin levels between $11.0 \mathrm{gr} / \mathrm{dl}$ and $11.9 \mathrm{~g} / \mathrm{dl}$ ), and $70.5 \%$ had hemoglobin levels between 7.0 $\mathrm{gr} / \mathrm{dl}$ and $10.9 \mathrm{~g} / \mathrm{dl}$. Conclusions: Anemia is highly prevalent in women of childbearing age in the native community Ese'eja Palma Real.

KEYWORDS: Anemia, iron deficiency, women's health, public health, prenatal care. (Source: MeSH NLM)

\section{INTRODUCCIÓN}

La anemia es un problema de salud pública a nivel global mayor en países en vías de desarrollo $(1,2,3)$. Según la OMS la incidencia a nivel mundial es aproximadamente de 2000 millones de personas (1). En América Latina la tasa promedio de anemia en mujeres de edad fértil es $20 \%$, variando de $8 \%$ en Chile y Uruguay a $35 \%$ en Guatemala, Cuba y Perú (4). La anemia aumenta el riesgo preconcepcional (5).

\footnotetext{
Facultad de Medicina Humana. Universidad Ricardo Palma, Lima, Perú.

2 Instituto de Investigación en Ciencias Biomédicas, Lima, Perú.Facultad de Medicina Humana. Universidad Ricardo Palma, Lima, Perú.

3 Hospital Central de las Fuerzas Aéreas del Perú. Lima, Perú.

a Interno de Medicina.

b Estudiante de pre grado.
} 
El Perú es un país pluricultural y multilingüe, con distintas etnias denominadas comunidades nativas. $\mathrm{La}$ comunidad nativa Ese Eja - Palma Real está localizada a orillas del río Madre de Dios, tiene alrededor de 300 habitantes, e incluyen familias provenientes del río Tambopata (Figura 1).

Los escasos estudios en comunidades nativas demuestran que la anemia en mujeres de edad fértil es alta, acompañándose de desnutrición y pobreza (6). Motivo por el cual el objetivo de nuestro estudio fue determinar la prevalencia de la anemia en mujeres en edad fértil en comunidades nativas Ese'eja en Madre de Dios.

\section{MATERIAL Y MÉTODOS}

Estudio observacional, descriptivo y transversal.

Se incluyeron a todas las mujeres de edad fértil (15-45 años) de la comunidad nativa Ese'Eja, 76/80 por censo interno de la población (7), sin controlar variables como embarazo, parasitosis, enfermedades hematológicas, menstruación; se excluyeron a las que no desearon participar en el estudio.

En vista que esta población es bilingüe (Takana/ Castellano), se contó con una técnica de enfermería propia de la comunidad Ese'Eja, en el equipo de trabajo, quien facilitó la comunicación en los casos que había dificultad para comunicarse en castellano.

Se contó con la autorización escrita de las Organizaciones Indígenas Nacionales, regionales y los jefes de cada comunidad, asimismo, se obtuvo el consentimiento informado escrito y firmado por cada uno de los participantes. Dentro de todo el proceso se respetaron las creencias y principios étnicos de la población amazónica. Se respetaron los principios éticos de acuerdo a la Declaración de Helsinki (8); se enmascararon los resultados y se entregaron al personal del centro de salud para la implementación de la terapia de suplementos de Hierro.

La recolección de las muestras se realizó durante la intervención MINSA-FAMURP en el mes de febrero del 2011, efectuadas en el Centro de Salud Clas III - Palma Real. Las muestras de sangre se obtuvieron por punción venosa, en tubos con heparina de sal de amonio como anticoagulante. Se realizó in situ la determinación inmediata del hematocrito, utilizando la centrífuga para micro-hematocrito (9). Para calcular el valor de la Hemoglobina, se dividió el valor del hematocrito entre tres.

Se definió anemia en mujer en edad fértil si la hemoglobina $(\mathrm{Hb})$ era menor de $12 \mathrm{~g} / \mathrm{dl}$. Se clasificó la anemia en: Severa si $\mathrm{Hb}<7,0 \mathrm{gr} / \mathrm{dl}$; moderada $\mathrm{Hb}$ entre 7,0 y 10,9 y leve si la $\mathrm{Hb}$ entre 11,0 y $11,9 \mathrm{gr} / \mathrm{dl}$, de acuerdo a los criterios de la OMS (2).

\section{Análisis de la información}

Los datos se ingresaron a una base elaborada en el programa Microsoft Access 2010 y se almacenaron en versión digital codificada en doble back-up. Posteriormente se realizó el control de calidad, y fueron analizadas con el paquete estadístico STATA 11.0 (STATA Corp. Texas, US).

Se utilizó estadística descriptiva y se obtuvieron frecuencias absolutas y relativas para las variables categóricas. Se evaluaron los supuestos de normalidad con el test de Shapiro-Wilk, y las medidas de tendencia central y dispersión en las variables numéricas.

\section{RESULTADOS}

La edad promedio fue $27,97 \pm 8,5$ años (rango: 16-45) el test de Shapiro-Wilk W fue 0,95 indicando normalidad (Gráfico 1).

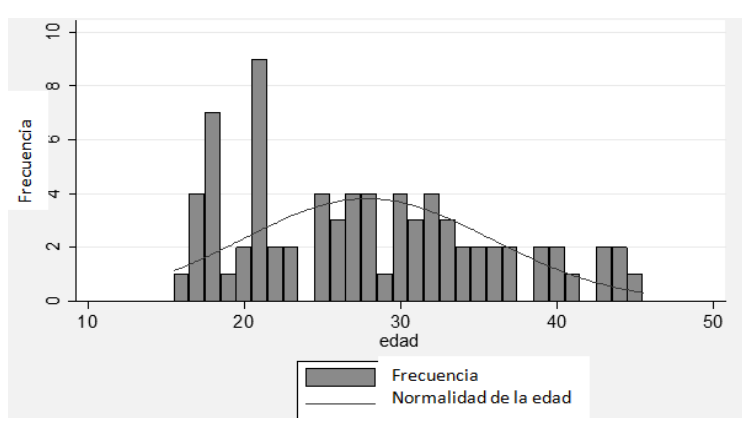

Gráfico 1. Distribución de edades de mujeres en edad fértil en la Comunidad Nativa Ese'eja Madre de Dios. 


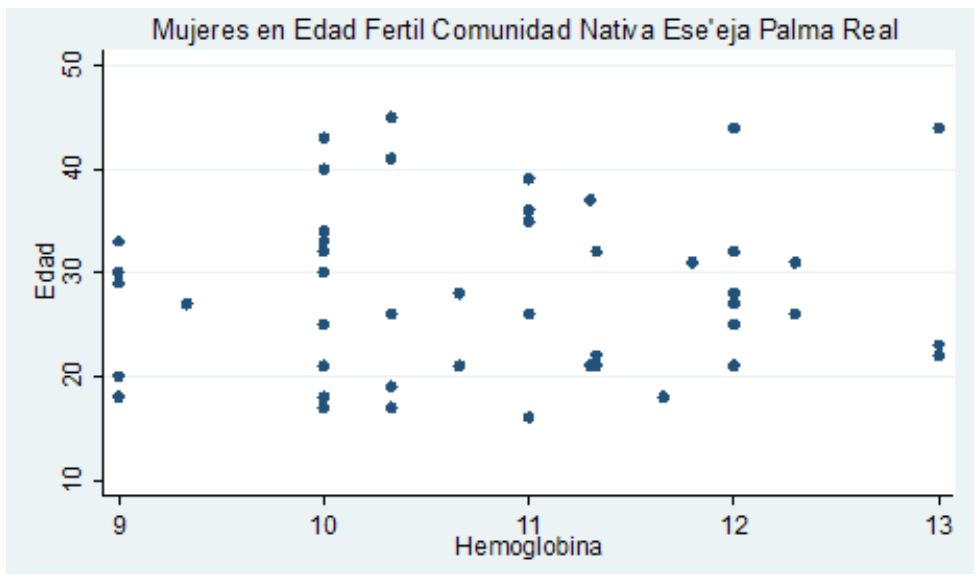

Gráfico 2. Niveles de Hemoglobina y edad en mujeres en edad fértil de la Comunidad Nativa Ese'eja Palma Real.

El nivel de hemoglobina promedio fue de 10,75 \pm 1,08 gr/dl (rango: 9,0-13,0). Cincuenta y nueve (77,63 \%) mujeres tenían hemoglobina $<12 \mathrm{~g} / \mathrm{dl}, 20 / 76$ $(29,5 \%)$ entre 11,0 y $11,9 \mathrm{~g} / \mathrm{dl}$, y el $70,5 \%$ restante entre 7,0 y $10,9 \mathrm{~g} / \mathrm{dl}$. No se encontraron mujeres con anemia severa.

No se encontró asociación estadística significativa entre edad y niveles de hemoglobina (Gráfico 2).

\section{DISCUSIÓN}

Los objetivos de este estudio fueron identificar la prevalencia de anemia, grados de anemia y niveles de hemoglobina en mujeres de edad fértil de las comunidades nativas Ese'eja Madre de Dios.

Se reportan niveles moderados de prevalencia de anemia en Latinoamérica (20,8\%), 24,8\%, 17,8$35,8 \%$ y $17,3-48,53 \%$ en México, Argentina y Cuba, respectivamente en contraste con lo encontrado en nuestro estudio (10-13).

El MINSA reporta una prevalencia de anemia en mujeres en edad fértil a nivel nacional de 28,0\% (14), mientras que diversos estudios reportan tasas de $18,1 \%$ de prevalencia general en Perú (10), 28,8\% en la selva baja (10), 35,26\% en Tambopata, Madre de Dios (14), y existe un reporte en la localidad de Pucallpa, con $70 \%$ de prevalencia en un hospital de la zona, este dato difiere grandemente de los anteriores y se explica por la alta prevalencia de parasitosis en la zona (15). En comunidades nativas, MINSA reporta 20,1\% (14) de prevalencia de anemia en mujeres en edad fértil; sin embargo nuestro estudio encontró $77,63 \%$, gran diferencia que amerita realizar futuras investigaciones en busca de factores asociados con la alta prevalencia en esta comunidad y probablemente en comunidades similares.

El nivel medio de $\mathrm{Hb}$ en mujeres en edad fértil en nuestro estudio $(10,75 \mathrm{~g} / \mathrm{dl})$ es inferior a los reportados en diversos países. En México un estudio similar describe un nivel medio de 13,1 g/dl (10), otro en Argentina 11,8 g/dl (12); e incluso un estudio similar en la selva baja $11,4 \mathrm{~g} / \mathrm{dl}$, motivo por el cual nos vemos obligados a continuar con las investigaciones en la comunidad para determinar factores predisponentes de esta patología, con el fin de elaborar políticas de salud para el desarrollo y prevención de programas disminuyendo la frecuencia anemia en estas comunidades.

Mientras que en nuestro estudio no se encontró asociación estadística significativa entre edad y niveles de hemoglobina, se ha reportado que la anemia presenta una tendencia decreciente conforme aumenta la edad, siendo de $23,7 \%$ para el rango entre 10 a 15 años y de $18,7 \%$ para el rango entre 36 a 45 años (14).

En conclusión, la prevalencia de anemia en mujeres en edad fértil de la comunidad nativa Ese'eja Palma Real es alta. Este es el primer reporte sobre el estado nutricional de estas comunidades.

\section{Fuente de financiamiento:}

Universidad Ricardo Palma

\section{Conflictos de interés:}

Los autores declaran no tener conflictos de intereses. 


\section{Correspondencia:}

José Antonio Grandez Urbina

Correo electrónico: jagrandez@gmail.com

\section{REFERENCIAS BIBLIOGRÁFICAS}

1. Organización Panamericana de la Salud. La anemia como centro de atención. Hacia un enfoque integrado para un control eficaz de la anemia. New York: Sesión conjuntadelaAsamblea Generaldelas Naciones Unidas y del Fondo de las Naciones Unidas a favor de la Infancia; 2004.

2. World Health Organization Iron deficiency anemia, assessment, prevention and control: a guide for programme managers. Geneva: WHO; 2001.

3. Davidson L, Nestel P. International Nutritional Anemia Consultative Group. Efficacy and effectiveness of interventions to control iron deficiency and iron deficiency anemia. Washington DC: International Life Sciences Institute; 2004.

4. Mora JO, Mora OL. Deficiencias de micronutrientes en América Latina y el Caribe: anemia ferropriva. Washington, DC: Organización Panamericana de la Salud; 1998.

5. Requeira J, Rodriguez $\mathrm{R}$, Brizuela $\mathrm{S}$. Comportamiento del riesgo preconcepcional. Rev Cubana Med Gen Integr. 1998; 14(2):160-164.

6. Huaman L. Estado nutricional y características del consumo alimentario de la población aguaruna. Amazonas, Perú 2004. Rev Peru Med Exp Salud Publica. 2006; 23(1): 10.

7. Instituto Nacional de Estadistica e Informática. Encuesta Demográfica y de Salud Familiar - ENDES 2010 (Internet).Lima, Perú: INEI; 2010 (Citado el 05 de junio del 2012). Disponible en: http://www1. inei. gob.pe/srienaho/Consulta_por_Encuesta.asp

8. Abajo F. La Declaración de Helsinki VI: una revisión necesaria, pero ¿suficiente? Rev Esp
Salud Pública. 2001; 75(5):407-420.

9. Sociedad Brasileña de Patología Clínica (SBPC)/ Medicina Laboratorial. Recomendaciones de la Sociedad Brasileña de Patología Clínica/ Medicina Laboratorial para la extracción de sangre venosa (Internet). ( $2^{\mathrm{a}}$ edición). Barueri: Editora Manole; 2010 (Citado el 05 de junio del 2012). Disponible en: http:// www.sbpc.org.br/upload/conteudo/ 320100928153008.pdf.

10. Gonzales G, Tapia V, Gasco M, et al. Hemoglobina materna en el Perú: diferencias regionales y su asociación con resultados adversos perinatales. Rev per med exp salud pública. 2011; 28(3): 484-491.

11. Shamah-Levy T, Villalpando S, Rivera JA, MejíaRodríguez F, Camacho-Cisneros M, Monterrubio EA. Anemia in Mexican women: a public health problem. Salud Publica Mex. 2003; 45(S4): S499-507.

12. Morasso M, Molero J, Vinocur P, et al. Deficiencia de hierro y anemia en mujeres embarazadas en Chaco, Argentina. Arch Latinoam Nutr. 2002; 52:336-43.

13. Gomis I, Gautier O. Deficiencia de hierro y ácido fólico en mujeres en edad fértil. Rev Cubana Hematol Inmunol Hemoter. 1998; 14(3):143-9.

14. Centro Nacional de Alimentación y Nutrición. Instituto Nacional de Salud. Ministerio de Salud. Anemia en gestantes del Perú y Provincias con comunidades nativas 2011. Lima, Perú: Instituto Nacional de Salud; 2012.

15. Becerra C, Gonzales GF, Villena A, de la Cruz D, Florián A. Prevalence of anemia in pregnancy, Pucallpa Regional Hospital, Perú. Rev Panam Salud Publica. 1998; 3:285-92.

Recibido: 03/09/12

Aceptado: 07/02/2013 